\title{
Calculation of collective modes for the Bose-Hubbard model with confinement
}

\author{
Emil Lundh \\ Department of Physics, Royal Institute of Technology, Albanova, SE-106 91 Stockholm, Sweden
}

\begin{abstract}
The collective excitations in the Bose-Hubbard model in a trap are studied by means of numerical diagonalization in one dimension. The strength function is calculated for monopole and dipole perturbations, and moments of the strength function are utilized in order to obtain information about the collective behavior under external forces. In the superfluid regime, the spectrum is found to be exhausted by one single frequency, while in systems that contain a Mott insulating plateau several frequencies are excited. An explanation of recent experimental findings in terms of a Mott plateau is suggested.
\end{abstract}

Recent years have seen a rapid development in the study of atoms confined in optical lattices. One of the virtues of such systems is that they provide an almost perfect realization of the bosonic Hubbard model. Corrections such as nearest-neighbor interactions are under control and can be made very small, and furthermore, the parameters of the model are widely tunable experimentally. In the case of spinless bosonic atoms the Bose-Hubbard model is known to support a Mott transition, and it has also been demonstrated experimentally by Greiner et al. [1] The addition of spatial confinement adds complexity to the well-known Bose-Hubbard model. In these inhomogeneous systems superfluid and Mott insulating phases may coexist in spatially separated regions 2]. However, a good experimental diagnostics is still lacking for the detection of this interesting type of state.

This paper wishes to forward the idea of using oscillatory modes in order to discriminate between different types of many-body state for these systems. Exciting oscillatory modes is an experimentally well-developed technique for studying the many-body physics of trapped condensates in the absence of optical lattices and has recently been applied also to the case with an optical lattice present 3, 4, 5]. Physical limits and basic dependences of the many-body eigenstates and energies were studied in an earlier paper 6] (cf. 7] ). Here we wish to relate the eigenvalue spectrum to the collective oscillations of the system and pose the physical question, what happens when the system is perturbed? Just as in Ref. [6], a five-particle system shall be studied using exact diagonalization, but the methods and concepts introduced here can be applied in numerical calculations using methods such as Monte Carlo, density-matrix renormalization group or mean-field calculations.

The system under consideration is a gas of bosonic atoms, confined in a harmonic trap superposed with an optical lattice formed by standing laser waves. By making the trap sufficiently tight in two directions, the system is effectively made one-dimensional, and when the optical-lattice potential is sufficiently strong the system is well described by the Bose-Hubbard Hamiltonian

$$
H=\frac{1}{2} U \sum_{r} a_{r}^{\dagger} a_{r}^{\dagger} a_{r} a_{r}-\sum_{<r r^{\prime}>} a_{r}^{\dagger} a_{r^{\prime}}+\frac{1}{2} \omega_{t}^{2} \sum_{r} r^{2} a_{r}^{\dagger} a_{r}
$$

The units are here chosen so that the tunneling strength is unity. $U$ is the on-site interaction strength, $\omega_{t}$ is the trap frequency, and $r$ is a dimensionless site index; the operators $a_{r}, a_{r}^{\dagger}$ destroy and create a particle at the site $r$, respectively, and obey Bose commutation relations. The sum subscripted $\left\langle r r^{\prime}\right\rangle$ runs over all pairs of nearestneighbor sites as usual.

We wish to know how the system responds to a perturbation of the type

$$
F=\sum_{r} v(r) a_{r}^{\dagger} a_{r}
$$

where $v(r)$ is a perturbing potential applied to the bosons. The strength function associated with the operator $F$ is [8]

$$
S(\omega)=\sum_{k \neq 0}|\langle k|F| 0\rangle|^{2} \delta\left(\omega-E_{k}\right),
$$

where $k$ labels the many-body eigenstates of the system and $E_{k}$ are the eigenenergies of the many-body system (in units where $\hbar=1$, and defining the ground-state energy as the zero level).

In the present exact diagonalization study, the strength function can be obtained exactly. However, considering the outlook of using numerical methods where larger systems can be treated, but the whole eigenvalue spectrum cannot easily be obtained, approximate methods must be considered. Reference [9] describes a moment method (cf. 10, 11]) that is well suited for describing the excitation spectrum for confined bosons in optical lattices. The moments of the strength function,

$$
m_{p}=\int d \omega S(\omega) \omega^{p},
$$

can, namely, be written as ground-state expectation values of commutators involving the Hamiltonian and the operator $F$. For the three moments that shall be used here, $m_{0}, m_{1}$ and $m_{2}$, one obtains

$$
\begin{gathered}
m_{0}=\left\langle 0\left|F^{2}\right| 0\right\rangle-\langle 0|F| 0\rangle^{2}, \\
m_{1}=\frac{1}{2}\langle 0|[F,[H, F]]| 0\rangle, \\
m_{2}=\langle 0|[F, H][H, F]| 0\rangle .
\end{gathered}
$$


The moment $m_{p}$ has the dimension of frequency raised to the $p$ th power, times the dimension of $F^{2}$, and therefore the quotients between different moments define frequencies: from the ones listed above we define $\omega_{10}=m_{1} / m_{0}$, $\omega_{20}=\left(m_{2} / m_{0}\right)^{1 / 2}$, and $\omega_{21}=m_{2} / m_{1}$. In the case where only one eigenstate is excited by $F$, these moment-related frequencies coincide and are equal to the frequency of that state, but more generally, they take on values between the frequencies of those modes that are appreciably excited by $F$, and the spread between them is related to the spread of the strength function. Observe that very little information about the individual excitation frequencies can be obtained from the moment calculation, except for the the fact that as $p \rightarrow-\infty$, the frequencies $\omega_{p, p-1}$ approach the lowest-lying eigenstate with a symmetry consistent with the perturbation $F[9]$. The three frequencies are, however, average excitation frequencies, in a sense that is made precise by considering their definition:

$$
\omega_{10}=\frac{\sum_{k \neq 0}|\langle k|F| 0\rangle|^{2} E_{k}}{\sum_{k \neq 0}|\langle k|F| 0\rangle|^{2}},
$$

and correspondingly for the other two frequencies. Furthermore, by writing $m_{0}$ as

$$
m_{0}=\sum_{k \neq 0}|\langle k|F| 0\rangle|^{2},
$$

it is clear that the zeroth moment is related to the probability for any excitation at all to happen, and it therefore gives information about the amplitude of the oscillations excited by the perturbation.

Let us now explore in the setting of an exactly diagonalizable system the relation between the strength function and the moment-related frequencies $\omega_{p q}$. The details of the diagonalization scheme were explained in Ref. [6]. The system consists of $N=5$ particles in one dimension subject to the Hamiltonian, Eq. (1). The perturbing potential is assumed to be on the form of Eq. (2) with the two choices of potential function

$$
\begin{aligned}
& v(r)=r \quad \text {, dipole perturbation (tilting), } \\
& v(r)=r^{2} \text {, monopole perturbation (compression). (8) }
\end{aligned}
$$

The exact strength function can be found by calculating the strength $S_{k 0} \equiv|\langle k|F| 0\rangle|^{2}$ for each excited state $k$. The strength function for the finite system is a sum of delta functions, and can of course not be plotted, but it is visualized in Fig. 1 by plotting a stem of height $S_{k 0}$ at the position $E_{k}$ of each excited state. For Fig. 1 the parameters are chosen as $\omega_{t}=0.3, U=0.1$, describing a weakly interacting system in a shallow trap, and the perturbing potential $v$ is chosen as the monopole perturbation. It is seen that only two almost-degenerate modes are excited with appreciable strength. These are the two lowest-lying monopole modes, $|2\rangle$ and $|3\rangle$, at $E_{2} \approx 0.5524$ and $E_{3} \approx 0.5776$; the excitation of the latter is weaker by approximately a factor 10 . The strength for the thirdstrongest excited state is weaker again by another factor

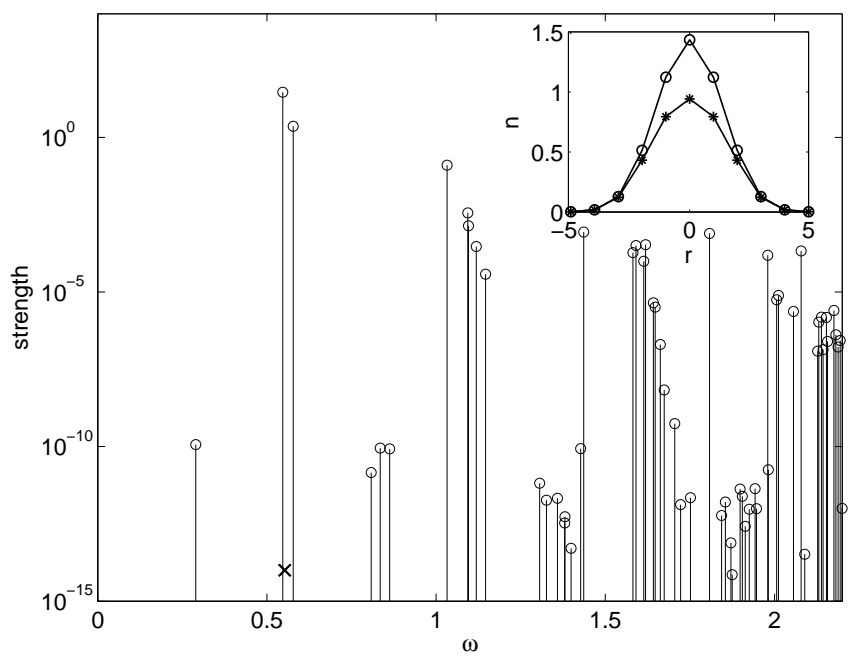

FIG. 1: Visualization of the strength function for a monopole excitation of a weakly interacting system, with $\omega_{t}=0.3, U=$ 0.1. The height of the stems represent the strength $S_{k 0}$ for each excited state $|k\rangle$, and their horizontal position indicates the frequency relative to the ground state of the excited state in question, $\omega=E_{k}$. The crosses mark the magnitude of three moment-related frequencies, in increasing order, $\omega_{10}, \omega_{20}$ and $\omega_{21}$. (However, the positions of the three crosses coincide completely in this figure.) The inset shows the density profile as circles and the quantum fluctuations in the local density as asterisks. All quantities are plotted in the dimensionless units employed throughout the paper.

20. The strengths for the eigenstates that have dipole symmetry are suppressed by about ten orders of magnitude, which corresponds to the numerical accuracy of the algorithm. This system is an almost pure Bose-Einstein condensate, its density resembling a discretized Gaussian, as seen in the inset of Fig. 1] Each of the system's collective modes is almost entirely exhausted by one many-body eigenstate or, as in the monopole case, an almost-degenerate pair of them.

Included in Fig. 1] as crosses, are the three momentrelated frequencies $\omega_{p q}$, which can be calculated using only ground-state properties. It is seen that all three frequencies lie close together - the symbols coincide almost completely so that only one is visible in the plot - and lie close to the main excitation frequency $E_{2}$, as expected. The magnitude of the zeroth moment is $m_{0}=30.6515$; this number makes more sense when compared to values of $m_{0}$ for other parameter choices. It can be roughly interpreted to mean that application of the potential $v(r)$, which means quadrupling the trap potential in this case, yields a population transfer rate of more than order unity to excited states and the oscillations should be clearly visible.

Figure 2 displays the results for the dipole excitation using the same parameters $\omega_{t}=0.3, U=0.1$. The picture is similar, but even simpler than in the monopole case: only one mode is appreciably excited, which in physical terms means that a perturbation with dipole 


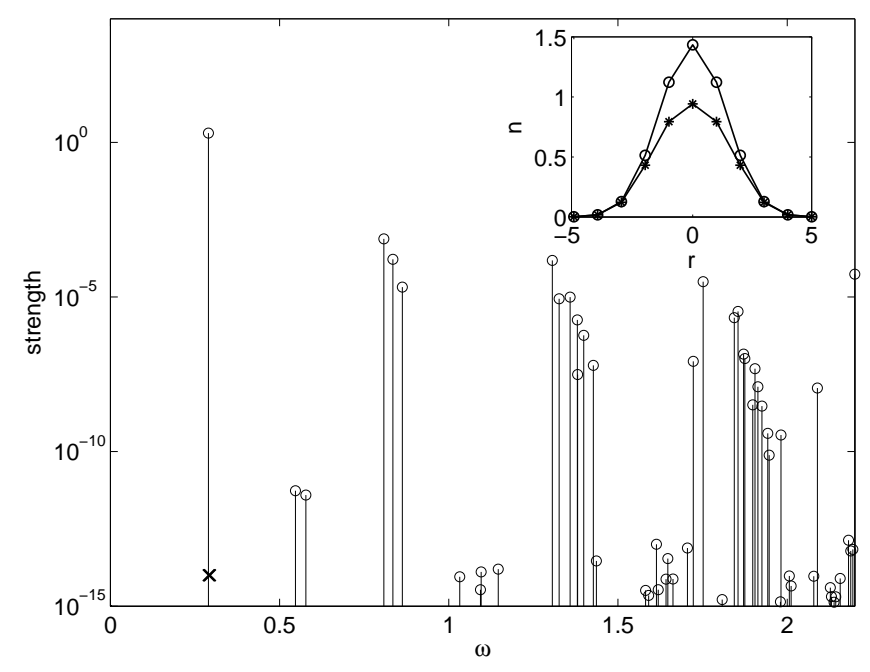

FIG. 2: Same as Fig. 1] but here the perturbation has dipole symmetry.

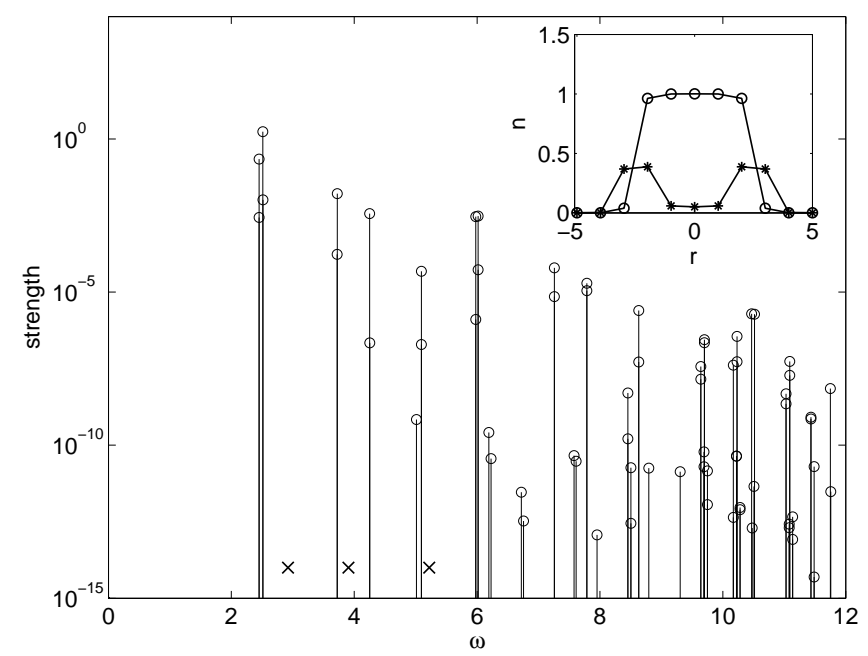

FIG. 3: Same as Fig. 1 but here the trap strength is $\omega_{t}=1$ and the coupling is intermediate, $U=20$. In the inset, the asterisks represent the quantum fluctuations in the density magnified by a factor 10 .

symmetry results in excitation of the lowest eigenstate of the many-body system, and thus oscillation at one welldefined frequency. The zeroth moment is $m_{0}=1.9928$, again of order unity.

Another example is shown in Fig. 3. The parameter choice $\omega_{t}=1, U=20$ corresponds to a mixed state with a rudimentary Mott insulating plateau in the center. At the present there is very little understanding of the oscillatory modes in this type of regime and little hope for an analytical approach. The results for the strength function is displayed in the main panel of Fig. 3 Clearly, the strength is now spread out over many eigenstates, although the largest strength is about a factor 10 larger than the next-largest. In experiment one can expect to observe a monopole oscillation with several frequencies, if

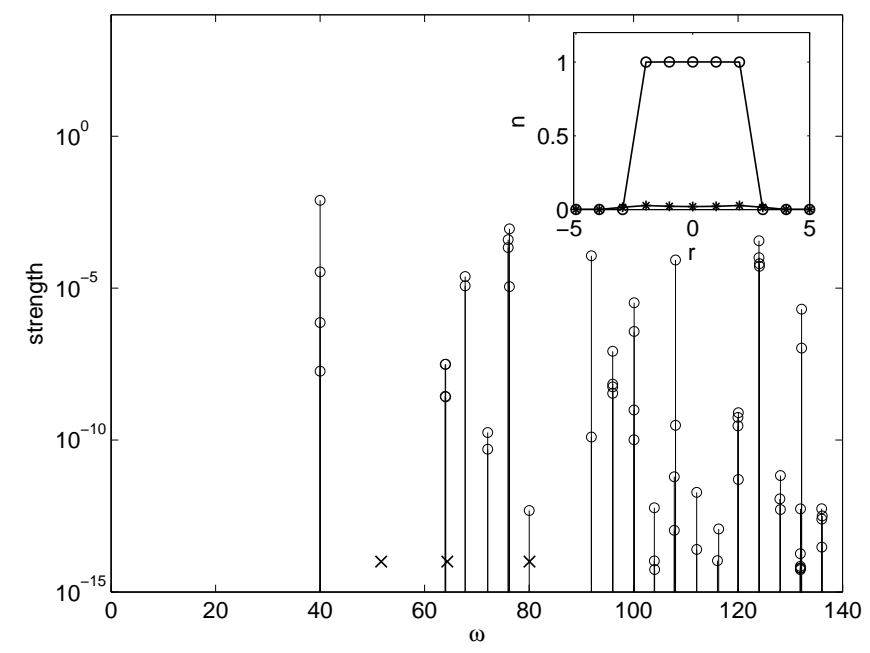

FIG. 4: Same as Fig. 11 but here the trap and the coupling are stronger, $\omega_{t}=4$ and $U=100$. In the inset, the asterisks represent the quantum fluctuations in the density magnified by a factor 100 .

damping is weak enough and the precision is good enough to resolve the different frequencies. The results of the moment calculation are likewise included in Fig. 3 The three moment-related frequencies now differ appreciably, as expected from the spread of the excitation strengths. As noted above, the moment calculation does not give direct information about the individual frequencies that are excited but the $\omega_{p q}$ lie between the frequencies of the most strongly excited modes. The zeroth moment for the Mott-plateau system is found to be $m_{0}=1.9596$. This indicates that again, excitation should result in visible oscillations.

The final example is in the limit of strong coupling in a tight trap, $U=100$ and $\omega_{t}=4$. This system is Mott insulating [6]. Figure 4 depicts the strength function and the moment-related frequencies for this system. Just as in the Mott-plateau case, the strength is distributed over several modes, which shows up as a difference between the moment-related frequencies. Observe, however, the magnitude of the strength function: correspondingly, the zeroth moment is now very small, $m_{0}=0.0094$. The reason is that the ground state for this system is a pure Fock state in configuration space, and therefore an eigenstate of the monopole operator and in fact of any operator of the form of Eq. (2), with very small corrections. This is of course a manifestation of the insulating nature of this state.

The final figure is an overview picture. Figure 5 displays the three moment-related frequencies in units of the lowest monopole frequency $E_{2}$; the zeroth moment $m_{0}$; and the quantum fluctuations in the local density at two points, all as functions of the coupling $U$ and the trap frequency $\omega_{t}$. The data has been recorded along an oblique path through phase space along the line $U=20 \omega_{t}$, where $\omega_{t}$ has varied from the value 0.3 up to 4 ; this way, all the 

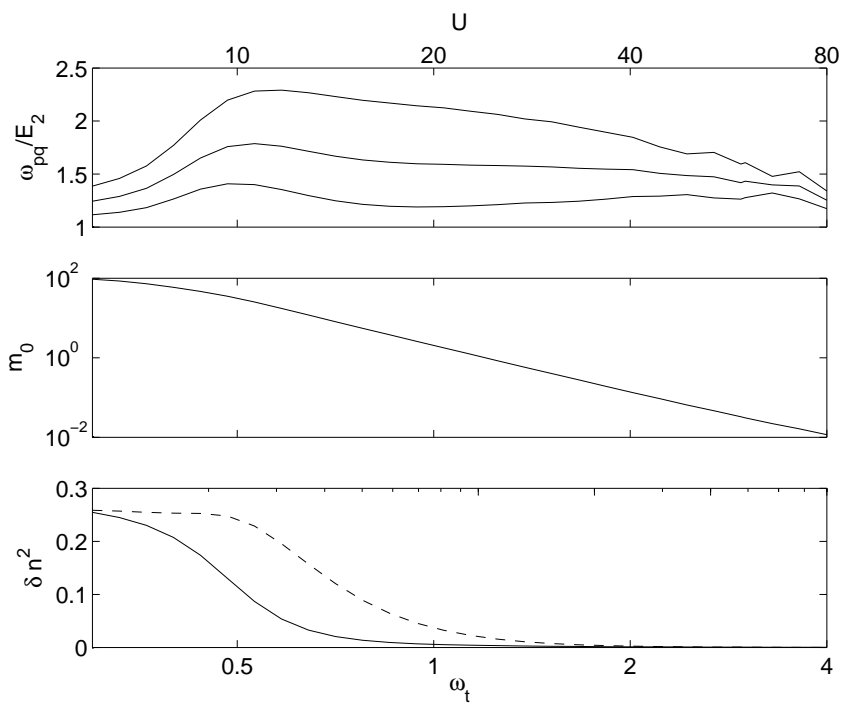

FIG. 5: Properties of the system as functions of the coupling $U$ and the trap strength $\omega_{t}$. The latter has been swept from $\omega_{t}=0.3$ to $\omega_{t}=4$, and and $U$ has varied as $U=20 \omega_{t}$ through the sweep. The topmost panel shows the three momentrelated frequencies (from top to bottom $\omega_{10}, \omega_{20}$ and $\omega_{21}$ ) in units of the lowest monopole frequency $E_{2}$. The middle panel shows the magnitude of the zeroth moment $m_{0}$. The bottom panel plots the quantum fluctuations in local density, $\delta n^{2}=\left\langle n^{2}\right\rangle-\langle n\rangle^{2}$, at the point $r=0$ (solid line) and $r=2$ (dashed line). The units are the dimensionless units employed throughout the paper.

phases discussed above could be sampled. For small $U$ and $\omega_{t}$, the system is superfluid and the density fluctuations are large. The moment-related frequencies stay close to each other and to the eigenfrequency $E_{2}$. The decrease of the density fluctuations in the centermost well signals the transition to a Mott-plateau state, and at the same time the frequencies begin to differ, but the zeroth moment is still above unity. The drop in $m_{0}$ comes at the same point as the drop in the fluctuations in the second off-center site; this marks the transition to the Mott insulating state. In this finite system, the Mott transition is gradual.

Reference [5] reports on an experiment that measures the response to excitation of trapped bosons in a threedimensional optical lattice. It is found that for a shallow lattice, which corresponds to a relatively small $U$ compared with the tunneling strength, one narrow frequency range is excited, just as expected for a trapped condensate. However, for larger $U$, a broad range of frequencies is excited with appreciable strength. This result, which is not easily interpreted in the framework of Bogoliubov theory, is in fact very similar to the present findings for a system with a Mott plateau. The drastic difference in size, dimensionality and method of excitation forbids quantitative comparison, but the qualitative similarities are there: we have seen in this paper that the rise and broadening of the frequencies is characteristic of being in the vicinity of the Mott-plateau regime.

In conclusion, the strength function for the monopole and dipole excitations of the Hubbard model in a trap have been calculated, and with an outlook to treating larger systems, the moments of the strength function have been employed in order to extract information about the excitation strengths and frequencies. It is seen in both the moment calculations and the full strength function how the finite system undergoes a transition between superfluid, Mott plateau, and fully Mott insulating states. It is predicted that the experimental signature of a system containing a Mott plateau is the excitation of several frequencies with appreciable strength, while a strongly coupled system will not be excited at all because of its Mott insulating nature. Based on the present findings, it is suggested that the broad and strong resonance found in a recent experiment [5] is due to a Mott insulating plateau in the system.

\section{Acknowledgments}

This project was financially supported by the Göran Gustafsson foundation.
[1] M. Greiner, O. Mandel, T. Esslinger, T. W. Hänsch, and I. Bloch, Nature 415, 39 (2002).

[2] D. Jaksch, C. Bruder, J. I. Cirac, C. W. Gardiner, and P. Zoller, Phys. Rev. Lett. 81, 3108 (1998).

[3] S. Burger et al., Phys. Rev. Lett. 86, 4447 (2002); F. S. Cataliotti et al., Science 293, 843 (2001).

[4] T. Stöferle, H. Moritz, C. Schori, M. Köhl, and T. Esslinger, Phys. Rev. Lett. 92, 130403 (2004).

[5] C. Schori, T. Stöferle, H. Moritz, M. Köhl, and T. Esslinger, e-print cond-mat/0408449 (2004).
[6] E. Lundh, Phys. Rev. A, in press.

[7] G. Pupillo, E. Tiesinga, and C. J. Williams, Phys. Rev. A 68, 063604 (2003).

[8] A. Bohr and B. R. Mottelson, Nuclear Structure (World Scientific, Singapore, 1998), Vol. 1.

[9] E. Lipparini and S. Stringari, Phys. Rep. 175, 103 (1989).

[10] G. M. Bruun, Phys. Rev. A 63, 043408 (2001).

[11] O. Bohigas, A. M. Lane, and J. Martorell, Phys. Rep. 51, 267 (1979). 